\title{
Situating Service Design: Approaches to embedding Service Design in Voluntary Community Sector Organisations
}

\author{
Dr Laura Warwick ${ }^{\mathrm{a}}$, Anna Spencer ${ }^{\mathrm{b}}$, Dr Angelika Strohmayer ${ }^{c}$, Dr Reem Talhouk ${ }^{\mathrm{d}}$ \\ a,c,d School of Design, Northumbria University \\ b Goldsmiths, University of London \\ * Corresponding author e-mail: laura.e.warwick@northumbria.ac.uk
}

\begin{abstract}
:
Interest in integrating Service Design in the Voluntary Community Sector (VCS) is growing. However, research regarding how Service Design is being situated within such organisations is limited. We conducted a series of 14 interviews with Service Designers in seven VCS organisations in the UK that varied in size and structure. Interviewees also varied in level of seniority within their design team. Our findings highlight the different approaches used to integrate Service Design(ers) into VCS organisations depending on: the variation in role; position in organisational hierarchy; and broader working contexts. We present the features of these approaches as a way of enabling future organisational contextualisation of practitioners' experiences. We hope to reduce barriers to organisational change needed for the successful integration of Service Design in VCS organisations.
\end{abstract}

Keywords: service design; embedding design; charities

\section{Introduction}

In the UK, many of the public services are provided by what is known as the Voluntary Community Sector (VCS). In 2017/18, the Government paid voluntary community organisations $f 15.7 \mathrm{bn}$ to deliver services on behalf of the state (NCVO, 2020). Over the last decade, the public service system has undergone a wholesale transformation to reduce costs, shift demand away from acute services, and deliver care that focuses on recovery and self-management (HM Government, 2010, 2011). However, these changes have been a "leap in the dark" (Gilburt, 2015) and with no prescribed approach to change for those designing or delivering services. Times of austerity have seen the sector strive to deliver more for less, and the far-reaching impacts of the pandemic have compounded this, with VCS organisations being a key component of emergency service delivery (Dayson, Baker and Rees, 2021).

In many circumstances, VCS organisations have looked to Design approaches to support their response to these external changes and help to secure their long-term survival in a turbulent context. However, there is little research that has explored how to position Design, particularly 
Service Design, in a VCS setting. With this paper, we follow on from some previous work that has shown the value of design to VCS (Warwick, 2015). Herein, we seek a deeper understanding of how service design has been embedded in the voluntary sector. Building on research with seven large and topically-diverse VCS organisations, we identify a series of distinguishing features between the approaches taken to embedding service design in VCS organisations, pointing towards some of the factors that influence the choice of approach.

We first present a short literature review about the challenges of embedding design in the VCS, followed by a description of our research approach and methods. After this, we present an overview of the three key features of the service design approaches used in the diverse set of organisations involved in the research. Finally we draw some conclusions about the potential implications of these features.

We do not aim to pit these approaches against one another with the aim of finding 'the best' approach to be used in VCS organisations. Rather, we present a plurality of features, each with their unique uses, reflecting the need for a multitude of approaches for this complex, diverse, and everchanging sector.

\section{Embedding Service Design in the Voluntary Community Sector}

The challenges of embedding service design (SD) in private sector organisations have been well documented (Kurtmollaiev et al, 2018; Bailey, 2012). Research has shown that when integrating SD into organisations, the value of design is not always acknowledged by all actors and there is a need for organisational cultural change (Wedin, 2019). In a recent survey with service design practitioners, Blomkvist and colleagues (2020) identified that organisational silos, culture, maturity, and willingness to engage in culture change as key challenges. Furthermore, confusion regarding the designers' expert identities within the organisation contributes to confusion about how their roles relate to the roles of their non-design colleagues (Blomkvist, Rodrigues \& Overkamp, 2020).

Even when SD is integrated into organisations, challenges remain in diffusing the approach across an organisation (Holmlid \& Malmberg, 2018) and in sustaining design capabilities (Seidelin et al, 2020). However, within large organisations, the push for the use of service design language, alignment of key performance indicators with service design objectives, and experimentation from senior management aid in overcoming these challenges (Kurtmollaiev et al., 2018). In contrast, Seidelin et al. (2020) found that for the successful integration of SD in Small Medium Enterprises (SMEs) a decentralised approach to engaging with SD was needed as it did not overburden a select group of employees with new commitments to the design process. Such a decentralised approach contributed to the sustainability of design capabilities, as employees across departments became curious about the activities of the decentralised team (Seidelin et al, 2020).

More specifically on embedding Service Design into VCS organisations, research has shown that not only should there be organisational readiness for integrating service design (Warwick, 2015), but individuals embarking on a service design journey within a VCS organisation should be given the time and space to do so, that is separate from their everyday practices of service delivery (Warwick, Pierri, Bradnam, \& Field, 2018). Furthermore, when exploring the integration of designers into VCS teams, it was highlighted that the view of the designer as an expert created a hierarchy that was a barrier to the co-creation process (Warwick et al., 2018). 
Despite a recent drive to integrate service design within VCS organisations' structures, there has been limited research exploring how VCS are embedding service design and service designers within their organisations. The aforementioned challenges faced by for-profit organisations also attempting to create a design culture highlights the importance of understanding how SD is being embedded in the VCS context.

\section{Research Process}

As the use of SD in VCS settings is a relatively new field of practice, this research sought the perspectives of embedded designers to gather their informed opinion on how the process could and should be used in this context.

Due to the relative immaturity of this field, there are only a small number of organisations with internal SD teams. To the knowledge of the researchers, at least one designer embedded in each VCS organisations with a SD function in the UK at the time of this research participated in the interviews. The sample was therefore able to saturate the pool of practitioners working in this way; a total of 14 participants. At the time of interview, or recently prior to taking part, all participants were designers embedded in a VCS organisation in a role entirely focused on SD. The sample included designers across the spectrum of seniority in their role within the embedded design team. The majority of participants were in delivery roles (nine), with a smaller percentage representing a managerial position (five).

These 14 participants worked across seven different VCS organisations in the UK for a minimum of 12 months - we present details of these in the table below (see Table 1). Most of the organisations in the sample are based in national charities with regional charities or offices associated with them.

Table 1. A table that details the organisations involved in the research and their ascribed pseudonym.

\begin{tabular}{|c|c|}
\hline Organisation and Interviews & Overview \\
\hline $\begin{array}{l}\text { A } \\
\text { Interviews } 1 ; 2 ; 3\end{array}$ & $\begin{array}{l}\text { This organisation, at its peak, had a team of four people } \\
\text { working between the national charity and its federation of local } \\
\text { charities to embed service design in their approach to work. } \\
\text { Their work focused on educating and supporting teams } \\
\text { designing new services. The first member of staff began in April } \\
2014 \text { in an unofficial design role and built up a team of service } \\
\text { designers. There remains one specific designer in the charity. }\end{array}$ \\
\hline Interviews $4 ; 5 ; 6 ; 7$ & $\begin{array}{l}\text { This organisation had an interdisciplinary team of seven with } \\
\text { three trained designers. The work focused on innovation and } \\
\text { organisational development in a national charity though did } \\
\text { also work with the charity's regional network. The first member } \\
\text { of staff began in May } 2013 \text { and recruited the team. The original } \\
\text { manager has now moved on and the team is currently in } \\
\text { transition. }\end{array}$ \\
\hline Interviews 8; 9; 10 & $\begin{array}{l}\text { This organisation has a design team of three. The work focuses } \\
\text { on both a national charity's core work as well as projects across } \\
\text { the network of local charities, specifically looking at user- } \\
\text { centred approaches to improving service delivery. The first } \\
\text { designer joined in October } 2014 \text { and has recruited a further two } \\
\text { designers, all of whom remain in the organisation. }\end{array}$ \\
\hline
\end{tabular}




\begin{tabular}{ll}
\hline D & $\begin{array}{l}\text { This organisation had designers embedded within a wider team } \\
\text { of researchers and developers. The full team was approximately } \\
\text { Interview } 11 \\
\text { strategic organisational development of a national charity and } \\
\text { also specific changes to tangible services and tools particularly } \\
\text { in the digital realm. }\end{array}$ \\
\hline E & This organisation had two staff employed as embedded \\
designers though only the interviewee had design training and \\
experience. The work focused on the improvement of services \\
within a national charity but had made limited tangible process \\
at the time of the interview. The interviewee was in post at the \\
time of the interview but has since moved on after one year in \\
the role.
\end{tabular}

The first author conducted 14 semi-structured interviews to allow for flexibility in the conversation with designers (Robson, 2011). These interviews were guided by a series of prompts, but ultimately were "discursive in nature" allowing the interviewees to "[raise] additional or complimentary issues" to guide and inform the direction of the research (Beardsworth and Keil, 1992: 261-2).

The interviews broadly covered the following topics:

- The designer's role in the VCS

- Motivation for working in the VCS

- Key differences about VCS work

- The value of design to the VCS

- How design should be implemented in VCS

- Main barriers and challenges for design and designers in VCS

- Future learning for design in VCS

We took a grounded theory approach to data analysis because of its serious commitment to 'representing all understandings' (Clarke, 2005: 3). The interviews were audio recorded and transcribed prior to three broad phases of data analysis. Firstly, initial coding (Charmaz, 2006) using an open and comprehensive approach to 'stick closely to data' and 'coding for actions' (Charmaz, 2014: 116) with an emphasis on designers' experiences. This was a deliberate attempt to reduce the researcher bias on data (Charmaz, 2014: 133). Secondly, a review for focused coding (Charmaz, 2006) where the most common codes and those offering most insight into the data were prioritised (Bryman, 2008: 543). And finally, a collaborative synthesis exercise to generate categories and start 
to identify some of the connections and relationships within the data. The final stage was undertaken by two researchers with complementary viewpoints; a service designer with direct experience of being embedded in a VCS organisation and a professional from a primarily VCS background. The outcome of the data analysis was 14 broad categories with 63 supporting clusters of codes.

Our analysis resulted in findings related to: barriers to success; impact of design; and community development, among others. However, in this paper we focus on the data related to the different ways that service designers are embedded in VCS organisations, as it lays the groundwork for future analysis, research and understanding within the field.

\section{Approaches to Embedding Service Design in VCS organisations}

Interviewees that were part of a founding service design team universally described being recruited without a clear service design strategy in place. In some cases, this was to purposefully design the strategy in the role (Organisation B and D), but in most cases, it was due to a lack of understanding of Service Design and the different ways in which it could be applied and grown in an organisation.

For this reason, this paper describes the approaches of embedding service design that have been detailed during these interviews. The analysis process has highlighted three notable features that provide distinction between the approaches: the roles of the designer; position in the organisational hierarchies; and broader working context. Each of these features is discussed below:

\subsection{Roles of the Service Designer}

There were three broad roles adopted by the embedded service designers in the data set: designer; advisor; and educator. The predominance of a role (or roles) can be seen as epitomising the service design strategy for the organisation e.g. a predominant educator role corresponds to a strategy of disseminating service design across the organisation.

The designer role can be viewed as a traditional design role, whereby the designer would lead and deliver the content of the design process, whilst working in collaboration with other teams within the organisation. In Organisation C, they described the team's predominant aim as working on 'discrete projects with discrete funding'. With this predominant strategy, the team is an internal design resource; in Organisation $D$, for example, every digital service had to be developed with the service design team.

The second role was that of advisor, where the design team would be consulted on projects happening elsewhere in the organisation and provide advice on how to conduct those using a service design approach. None of the organisations had this as a predominant strategy, but it was adopted alongside other roles as a way of dealing with demand (Organisation A) and ensuring the quality of service design applied by non-designers (Organisation B).

The third and final role was educator, which focused on upskilling stakeholders in service design. Within this role, the data showed two distinct approaches: educating a few selected people for the purposes of becoming part of the extended design team (as in Organisation D); or training staff in service design tools and methods to increase awareness and skills level across the organisation (as in Organisation $A$ and $E$ ).

None of the designers interviewed described having a singular role. More often than not, they adopted all three roles at different times and in different contexts. However, in some organisations, taking on the additional roles was not part of the design strategy. For example, in Organisations $F$ 
and $\mathrm{G}$, interviewees describe people being upskilled as a result of being involved in the design process led by the design team, rather than taking on the role of an explicit educator. In Organisation $B$, they describe a change in the organisational strategy from central decision-making, to regional control. This prompted a shift from peoples' dominant roles as 'designers', to adopting the role of 'advisors'.

\subsection{Position in organisational hierarchy}

Another feature of the embedded service design approach is the position the designers' hold in the organisation's hierarchy.

Only one of the organisations interviewed, Organisation B, had a member of staff who was part of the executive management team. Designer $X$ reflected that this was 'lucky' as it allowed them to 'open doors' and say: "Hello! This is what we're doing. You should really be doing things differently". In the other cases, the lack of extensive executive buy-in impeded the impact of service design within the organisations. The designer in the executive management team stated that:
"I think the service design at [Organisation C] almost happened by accident with our leadership in the organisation, not knowing what it was or knowing that they wanted it or needed it, and that's what's really slowed down how fast you can start making an impact, and it's made it quite a challenge to embed service design into your organisation"

The interviewees reflected that an 'ideal' design team would have a member of staff that was involved in Executive management meetings to more meaningfully integrate the organisational strategy and activity with that of the service design team.

The majority of the designers interviewed described the most senior designer as being part of midlevel management. Depending on the layers of management above them, this meant that they could be quite far removed from decision-making discussions. Designer X in Organisation C said: "there was no drive from a director level... we worked on projects as they came along. There was no overall strategic view of what should happen to our team."

Only one organisation, Organisation F, described purposefully positioning the service designer in a frontline delivery team. This was mainly because they were supporting a focused piece of work as an in-house design resource. Organisation E had two junior service designers, who should have been managed by a senior service designer positioned in middle-management, but the role was not filled and subsequently removed.

\subsection{Broader working context}

The third and final feature of the embedded service design approaches is the broader working context in which the design team operates.

The service design teams in the data set were aligned with one of three departments: digital (Organisations D and G); business development (Organisation C); or service development/delivery (Organisations A, B, E and F). In all cases, this was related to the position of the person in the organisation who had shown the initial interest in service design, rather than necessarily being a conscious choice about where best to situate the team.

Any of these locations (or another) could be the right one for the service design team depending on the SD strategy employed. However, analysis showed that the impact of the team was impeded when the rest of the department advocated a conflicting approach to the tenets of SD. In Organisation $B, D$ and $F$, the wider team are all adopting the same set of service design principles in 
their work and they feel this helps them to be understood and spread service design practice. Designers in Organisation $C$ talked about repositioning their team in the next 6 months to also achieve this alignment, seeing it as a way to increase their reach and impact. In contrast, Organisation A, E and $\mathrm{G}$ talked about instances where their approach clashed with the principles of the wider team. The designer in Organisation A said this resulted in them tempering their approach: "I feel like I've really had to pick my battles... I want to rock the boat, but I want to stay in it".

\section{Conclusions}

The approaches of embedding service design that are presented in this paper are not intended to be interpreted as blueprints for the VCS. Instead, they should be seen as prompts for more conscious decision-making on the three key features of the approaches: the (predominant) roles of service designers; the position in the organisational hierarchy; and the broader working context (see Table 2).

Table 2. A table that summarises the approaches' key features.

\begin{tabular}{ccc}
\hline Roles of the Service Designer & $\begin{array}{l}\text { Position in Organisational } \\
\text { Hierarchy }\end{array}$ & Broader Working Context \\
\hline - Designer & $\bullet$ Executive & $\bullet$ Digital \\
- Advisor & $\bullet$ Middle Management & $\bullet$ Business \\
- Educator & $\bullet$ Frontline Delivery & development \\
& & Service \\
& & development/ \\
& & delivery \\
\end{tabular}

These features should be considered in the context of the overall ambition for Service Design within the organisation. If the aim is to create a diffused design culture (Manzini, 2015) in the organisation, then executive leadership is a necessity. Similarly, positioning the service design team within a department that aligns with the principles of the work can amplify the impact of the team. Without this alignment and senior-level representation, the team can only act as a limited design resource. Importantly, there is also a need to adopt the principles of service design when creating the team; prototyping the approach and having permission to change and reposition it would help to maximise its potential impact. Similarly, continuing to iterate the approach ensures that it reflects the changing needs of the organisation.

We hope that knowledge of these distinctive features will help service designers have more useful conversations about their potential contribution to the VCS. Furthermore, that VCS organisations will be able to integrate service design in a way that meets the needs of their context, without hampering the impact of the approach, ensuring value is achieved for finite resources at this time of continuing uncertainty for the sector and its beneficiaries.

\section{Further Research}

There is much learning from this research that we hope to share in future publications. A limitation of this study is that it has focused on the service designers' perspective on their work. Further research that gathered additional organisational perspectives, particularly of management and 
budget holders, would greatly add to our understanding of the particular benefits and challenges of seeking to embed SD in a VCS context.

\section{References}

Bailey, S. (2012) Embedding service design: the long and the short of it, in Proceedings of ServDes. 2012. Third Nordic Conference on Service Design and Service Innovation. Espoo, Helsinki: Laurea University of Applied Sciences, pp. 1-11.

Beardsworth, A. and Keil, T. (1992) The Vegetarian Option: Varieties, Conversions, Motives and Careers, Sociological Review, 40: 253-93.

Blomkvist, J. Rodrigues, V and Overkamp, T. (2020) Challenges facing service design practitioners: a pilot study, in Proceedings of the ServDes.2020 Conference. Melbourne, Australia. Retrieved 5 May, 2021, from http://servdes2020.s3.amazonaws.com/uploads/event/paper/74/101_Blomkvist_Rodrigues_Over kamp_SP.pdf

Bryman, A. (2008) Social Research Methods, Third Edition, Oxford: Oxford University Press

Charmaz, K. (2006) Constructing Grounded Theory: A Practical Guide through Qualitative Analysis, London: Sage

Charmaz, K. (2014) Constructing Grounded Theory, Second Edition, London: Sage

Clarke, A.E. (2005) Situational Analysis - Grounded Theory After the Postmodern Turn, London: Sage Publications.

Dayson, C., Baker, L. And Rees, J. (2021) The Value of Small in a Big Crisis, Lloyds Bank Foundation, London. Retrieved 5 May, 2021, from

https://www.lloydsbankfoundation.org.uk/media/cqhjhftd/lbfew-value-of-small-2021-summaryreport.pdf

Gilburt H. Mental Health Under Pressure. London: The King's Fund. Retrieved 5 May, 2021, from http://www.kingsfund.org.uk/publications/ mental-health-under-pressure

Holmlid, S., \& Malmberg, L. (2018). Learning to design in public sector organisations: A critique towards effectiveness of design integration. ServDes2018. Service Design Proof of Concept, Proceedings of the ServDes.2018 Conference. Milano, Italy. Retrieved 5 May, 2021, from http://www.servdes.org/wp/wp-content/uploads/2018/07/4.pdf

HM Government. (2010). The Coalition: our programme for government. London. Retrieved 5 May, 2021, from

https://www.gov.uk/government/uploads/system/uploads/attachment_data/file/78977/coalition _programme_for_government.pdf

HM Government. (2011). Open Public Services White Paper. Retrieved 5 May, 2021, from https://assets.publishing.service.gov.uk/government/uploads/system/uploads/attachment_data/f ile/255288/OpenPublicServices-WhitePaper.pdf

Kurtmollaiev, S., Fjuk, A., Pedersen, P., Clatworthy, S., \& Kvale, K. (2018) Organizational Transformation Through Service Design: The Institutional Logics Perspective. Journal of Service Research, 21(1), 59-74. Retrieved 5 May, 2021, from https://doi.org/10.1177/1094670517738371

Manzini, E. (2015). Design, When Everbody Designs. Cambridge, USA: MIT Press.

NCVO (2020) UK Civil Society Almanac 2020. Retrieved 19 May, 2021, from: https://data.ncvo.org.uk/about/

Robson, C. (2011). Real World Research (3rd Edn.). Chichester, Sussex: John Wiley \& Sons.

Seidelin, C. Sivertsen, S. M. and Dittrich, Y. (2020) Designing an organisation's design culture: How appropriation of service design tools and methods cultivates sustainable design capabilities 
in SMEs, in Proceedings of the ServDes.2020 Conference. Melbourne, Australia. Retrieved 5 May, 2021, from http://www.ep.liu.se/ecp/173/001/ecp20173001.pdf

Warwick, L. (2015) Can design effect transformational change in the voluntary community sector? [Doctoral thesis, Northumbria University]. Northumbria Research Link. http://nrl.northumbria.ac.uk/24459/

Warwick, L., Pierri, P., Bradnam, C., \& Field, E. (2018). Funding Service Design: growing Service Design practice through a grants programme. Paper presented at ServDes.2018 - Service Design and Service Innovation conference 2018, Milan, Italy.

Wedin, J. (2019). The State of Design - Swedish Edition 2019. Retrieved 19 May, 2020, from https://medium.com/@jenswedin/the-state- of-design-swedish-edition-2019-by-designleadership-sweden- d1b98ceb147b

Author Bios:

Dr Laura Warwick is a Senior Lecturer in Service Design and Social Innovation at Northumbria University. She works with local and national voluntary organisations to support them to use Service Design to improve their services and systems.

Anna Spencer is a doctoral student at Goldsmiths, University of London based in both Social, Therapeutic and Community Studies and the Department of Design. Her research focuses on the intersection between social design and community work.

Dr Angelika Strohmayer is a Senior Lecturer and co-director of the Design Feminisms research group at Northumbria University's School of Design, co-editor of the Meaningful Design Processes Forum in Interactions Magazine and founding member of fempower.tech.

Dr Reem Talhouk is a Research Fellow at Northumbria University in the School of Design and the Centre for International Development. She is the co-director of the Design Feminisms Research Group and the 2020 Young Digital Leader. 